\title{
Averaging of Temporal Memories by Rats
}

Dale N. Swanton
Villanova University

\author{
Cynthia M. Gooch \\ University of Pennsylvania School of Medicine
}

\author{
Matthew S. Matell \\ Villanova University
}

\begin{abstract}
Rats were trained on a mixed fixed-interval schedule in which stimulus A (tone or light) indicated food availability after $10 \mathrm{~s}$ and stimulus B (the other stimulus) indicated food availability after $20 \mathrm{~s}$. Testing consisted of nonreinforced probe trials in which the stimulus was $\mathrm{A}, \mathrm{B}$, or the compound $\mathrm{AB}$. On single-stimulus trials, rats responded with a peak of activity around the programmed reinforced time. On compound-stimulus trials, rats showed a single scalar peak of responding at a time midway between those for stimulus A and B. These results suggest that when provided with discrepant information regarding the temporal predictability of reinforcement, rats compute an average of the scheduled reinforcement times for the A and B stimuli and use this average to generate an expectation of reward for the compound stimuli.
\end{abstract}

Keywords: peak-interval procedure, interval timing, compound stimulus, vector averaging, time perception

Supplemental materials: http://dx.doi.org/10.1037/a0014021.supp

The ability of organisms to behave in accordance with the temporal relationships between events in the world is important for survival (Bateson, 2003; Brunner, Kacelnik, \& Gibbon, 1992), and has recently been proposed to play a central role in associative learning (Gallistel \& Gibbon, 2000; Kirkpatrick \& Church, 2000; Miller \& Barnet, 1993). Timing in the seconds to minutes range, interval timing, has been demonstrated in a wide range of species, ranging from honeybees to humans (Boisvert \& Sherry, 2006; Henderson, Hurly, Bateson, \& Healy, 2006; Rakitin et al., 1998), and shows characteristic features such as flexibility (i.e., animals are able to time stimuli that have randomly occurring onsets) and relativity (i.e., the magnitude of errors is directly proportional to the interval being timed).

To account for this perceptual and behavioral capacity, models of interval timing generally consist of three components; a clock, memory, and decision process (Church, 1997). Briefly, the clock process provides some representation of the amount of time that has elapsed since an event (e.g., a linear accumulator), the memory process stores the current clock representation whenever a biologically relevant event takes place (e.g., on reinforcement), and the decision process determines whether the current representation of elapsed time is sufficiently close to a memory of past event times to warrant responding in a "time's up" manner. As such, interval

Dale N. Swanton and Matthew S. Matell, Department of Psychology, Villanova University; Cynthia M. Gooch, Department of Cognitive Neurology, University of Pennsylvania School of Medicine.

We are indebted to Chara Malapani for pointing out the possibility of these results during a presentation in October 2006.

Correspondence concerning this article should be addressed to Matthew S. Matell, Department of Psychology, Villanova University, 800 Lancaster Avenue, Villanova, PA 19085. E-mail: matthew.matell@villanova.edu timing models correctly predict that timing-related behavior will, on average, reflect the temporal aspects of past experience. Further, even in situations in which the programmed duration varies in a systematic manner across trials (e.g., the time of reinforcement is progressively later on each trial), the addition of a weighting parameter that reflects the recency of the temporal memory can account for the finding that animals can track the systematic change (Higa, 1997).

However, it remains unclear what occurs when an animal is presented with a stimulus set that offers conflicting information about the possible time of reinforcement. For example, if one signal indicates that reinforcement may be available after a short delay, but another signal indicates that reinforcement may only be available after a long delay, what is the appropriate response when both signals are presented together? One conceivable option would be to "cover-both-bases," and respond at the early expected time and if reinforcement does not arrive, then respond again, or continue responding, until the later expected time. In this manner, the animal would be certain to obtain reinforcement as soon as it became available. In fact, previous findings from classical conditioning studies, using "stimulus compounding" (Kehoe, 1986), suggest a summation of responding when two individually trained excitatory stimuli are presented together (Emurian \& Weiss, 1972; Miller, 1971; Weiss, 1972). Although summation is not a universal finding (see McLaren \& Mackintosh, 2002, for a review), evidence suggests that when the two stimuli come from different modalities, as in the current report, summation is more reliably observed (Aydin \& Pearce, 1997). An alternative possibility is that the animal responds at either the early or late duration, but not both. This either/or strategy may result in missed food reinforcement, but it allows other behaviors to be pursued at a higher rate. This option might be expected from previous work showing a biased selection of a temporal memory at the beginning of the trial based 
on stimulus probability or predictive cues (Meck, 1984), selection of the shorter of two sampled memories when multiple cues are provided (Meck \& Church, 1984), and behavior controlled by temporal distance (Fairhurst, Gallistel, \& Gibbon, 2003). Finally, a third alternative would be to combine, in some manner, the discrepant information, and respond at a time that is in between the two expected outcomes. This option might be expected given work showing spatial, temporal, spatial-temporal, and circadian timeinterval time vector averaging in pigeons (Cheng, 1989; Cheng \& Roberts, 1991; Cheng, Spetch, \& Miceli, 1996; Gibbon, Fairhurst, \& Goldberg, 1997).

The present experiment addressed this question using a mixed peak-interval procedure, in which rats were trained that reinforcement could be earned for nosepoking after a short duration (10 s) following the presentation of one modal stimulus (e.g., a tone), or it could be earned after a long duration (20 s) following the presentation of a different modality stimulus (e.g., a light). On a subset of nonreinforced probe trials, we presented both signals in compound and found that the rats computed an average of the two durations held in temporal memory, and then timed this averaged duration in an approximately normal manner.

\section{Method}

\section{Subjects and Apparatus}

Ten adult male Sprague-Dawley rats (Rattus norvegicus; Harlan, Indianapolis, IN) approximately 3 months of age at the beginning of the experiment were used. Rats were housed in pairs with a 12-hr light-dark cycle (lights on at 8 a.m.). All behavioral testing was conducted during the light phase. The rats had ad libitum access to drinking water, but were kept on a restricted feeding schedule (Harlan 2019 Rat Diet) to maintain their body weights at 85 to $90 \%$ of free-feeding levels, adjusted for growth. Rodent chow was provided immediately following each daily session.

Training and testing took place in 10 standard operantconditioning chambers $(30.5 \times 25.4 \times 30.5 \mathrm{~cm}$, Coulbourn Instruments, Allentown, PA). The sides of the chamber were ventilated Plexiglas, and the front and back walls and ceiling were aluminum. The floor was composed of stainless steel bars. A pellet dispenser delivered 45-mg sucrose pellets (Formula F; Noyes Precision, Lancaster, $\mathrm{NH}$ ) to a food magazine on the front wall of the chamber. Three nosepoke response apertures $(2.5 \mathrm{~cm}$ opening diameter) with photobeam detection circuits were placed on the back wall of the chamber, and had yellow and green LED cue lights in their interior. Aluminum "hallways" $(30.5 \mathrm{~cm}$ high $\times 8.2$ $\mathrm{cm}$ deep) were attached to the back wall in between the nosepoke apertures to limit the quantity and rapidity of behavioral switching between nosepoke apertures. The operant chambers were equipped with a houselight and a seven-tone audio generator. Stimulus control and data acquisition were achieved using a standard operant-conditioning control program (Graphic State, Coulbourn Instruments), with a temporal resolution of $20 \mathrm{~ms}$.

\section{Procedure}

The rats progressed through a sequence of nosepoke training, fixed-interval training with two stimuli/durations, peak-interval training with two stimuli/durations, and peak interval testing, which was identical to the peak-interval training stage, but with the addition of compound stimulus presentation on a proportion of the probe trials. Rats were run 5 days per week at the same time each day. Two days before training commenced, rats were given 20 , 45-mg sucrose pellets in their home cage.

Nosepoke training (two to six sessions). Rats were given a minimum of two sessions of magazine training, in which a sucrose pellet was delivered on a fixed ratio 1 schedule on the center nosepoke aperture, until 60 reinforcers had been earned or $2 \mathrm{hr}$ elapsed. To prevent the nosepoke apparatus from delivering multiple reinforcers per entry into the nosepoke aperture, a 2-s "timeout" was instituted following the delivery of each reinforcer. Rats were maintained on this training procedure until they met the 60 reinforcer criterion on two sequential sessions.

Fixed-interval training (five sessions). Trials commenced with presentation of either the 10-s "short" stimulus (either a $4 \mathrm{kHz}$ steady tone or illumination of the houselight; counterbalanced) or the 20-s "long" stimulus (the other stimulus). The first nosepoke entry into the center nosepoke aperture after the associated criterion duration elapsed was reinforced and the stimulus terminated. Responses made prior to the criterion duration had no programmed consequence. A variable, uniformly distributed 30 to $70 \mathrm{~s}$ intertrial interval (ITI) separated trials. Sessions lasted $2 \mathrm{hr}$ in this and the subsequent procedures.

Peak-interval training (52 to 55 sessions). Peak-interval training was identical to fixed-interval training, except that nonreinforced probe trials were presented in addition to the reinforced trials. Probe trials were identical to the fixed-interval trials, except that the trials terminated independently of responding at a duration that was 2.5 to 3.5 times the criterion duration for that signal (e.g., "short" probe trials lasted 25 to $35 \mathrm{~s}$ ) and no reinforcement was provided. To equate reinforcement density between the two signals, $25 \%$ of the short signal trials were reinforced, whereas $50 \%$ of the long signal trials were reinforced. Trial type was selected at random with replacement.

Peak-interval testing (15 sessions). Peak-interval testing was identical to peak-interval training, with the addition of compound stimulus probe trials on $25 \%$ of the trials. On these compound probes, both the $4 \mathrm{kHz}$ tone and the houselight commenced simultaneously, and then terminated without reinforcement in a response-independent manner after 50 to $70 \mathrm{~s}$.

\section{Analysis}

Mean functions. The mean response rate as a function of signal duration on probe trials was computed using 1-s bins. The data from each rat were pooled into five session blocks to minimize variability in single session responding due to the low numbers of probe trials. The resulting distribution, referred to as a peak function, relates the probability of responding to the subjective estimate of time passed. To obtain quantitative indexes of these response functions, each distribution was fit with a five parameter Gaussian function: $\mathrm{a} * \exp (-1 / 2) *((\operatorname{abs}(\mathrm{x}-$ b)/c) d) + e. The first two parameters relate to the amplitude, and the mean. The third parameter relates to the width, whereas the fourth parameter enables better fit by accounting for kurtosis, and the fifth parameter accounts for a baseline rate of responding. Peak rate was taken as (a). Peak time was taken as (b). Peak spread was computed as the width of the function at 
half maximum by: $2 * c *\left((2 * \log (2))^{\wedge}(1 / d)\right.$. The normalized spread was computed as peak spread/peak time.

Single trials. Single trial analyses were performed by characterizing the temporal pattern of responses on each trial as a two state, low-high-low, step function as described in detail previously (Church, Meck, \& Gibbon, 1994; Wiener, Magaro, \& Matell, 2008). Briefly, this entailed performing an exhaustive iterative fitting procedure to minimize the absolute deviations between a single step function and the response times on each trial. The data obtained from these fits is the time at which the response rate abruptly transitions from a low rate to a high rate (start), and the time at which it transitions from a high rate back to a low rate (stop). As has been demonstrated previously (Matell, Bateson, \& Meck, 2006), trials with low numbers of responses show very poor evidence of temporal control (i.e., their mean response distributions are flat). Further, as a result of their low response rate, they produce little impact on the shape and placement of the mean response function. In contrast, these low response trials are weighted equally with trials that have many responses when single trial statistics are used. As such, only those single trials with at least three responses, and step functions wider than $2 \mathrm{~s}$, were used in analyses based on step function fits. This requirement left $63 \%$, $88 \%$, and $82 \%$ of the $10-\mathrm{s}$, compound, and 20 -s trials, respectively, for analysis. Although this differed between trial types, $F(2,16)=$ $31.297, p<.001$ due to a smaller percentage of 10-s trials as compared to both compound and 20-s trials (both $p<.001$ ), we feel that the use of this restriction leads to a better reflection of temporally controlled responding than allowing trials with single responses to contribute equally.

Statistics. Peak times, peak rates, and normalized spreads from the fitted mean functions, and the start and stop times, as well as their coefficients of variation, from the single trial analyses were separately analyzed using a repeated measure analysis of variance (ANOVA), with duration (10-s stimulus, compound stimulus, 20-s stimulus) as a within-subject factor, and modality (short light/long tone, short tone/long light) as a between-subjects factor. Our initial analyses were done with the data obtained during Block 1 only, to examine the nature of responding before the rats had extensive exposure to the nonreinforced compound stimulus. Following these analyses, we evaluated whether temporal responding changed by conducting an additional set of ANOVAs with the addition of block (3 levels) as a within-subject factor. LSD post hoc tests were used to probe significant effects. In cases in which sphericity was violated, the Greenhouse-Geiser correction was used for significance testing.

\section{Results}

Average peak functions from Block 1 are presented in Figure 1A. As can be seen, response rate peaked around the criterion times of $10 \mathrm{~s}$ and $20 \mathrm{~s}$ during probe trials with the short and long stimuli alone. Similarly, responding during compound trials was peak shaped, and fell in between the peak functions for the single cue trials. Compound responding was roughly scalar as superimposition can be seen (with the exception of the right tails) when the peak functions are plotted in terms of relative duration (Figure 1B).

An ANOVA for peak times from Block 1 confirmed the difference in responding across durations, $F(2,16)=122.990, p<.001$. Post hoc pairwise comparisons indicated that peak times on the compound cue were significantly different from the peak times on both the 10-s and 20-s cues alone (both $p<.001$ ). An ANOVA for peak rates revealed a significant effect of duration, $F(2,16)=$ $3.854, p<.05$ with post hoc analyses indicating that peak rate during the compound cue (.961 resp/s) was higher than that during the 10 -s cue $(.692 \mathrm{resp} / \mathrm{s} ; p<.05)$, but not different from the 20 -s cue (.842 resp/s). There was also a significant effect of duration for normalized spreads, $F(2,16)=6.662, p<.05$. Pairwise comparisons, however, revealed that this difference resulted from tighter relative responding on the 20 -s cue (.960) as compared to both the 10 -s (1.054) and compound cues (1.071; both $p<.05)$, which did not differ from each other. There were no main effects of stimulus modality, $F(1,8)=0.292,3.022,0.079$ for peak rate, peak time, and normalized spread, respectively, nor any Modality $\times$ Duration interactions, $F(2,16)=1.781,2.457,1.614$ for peak rate, peak time, and normalized spread, respectively, for these mean function statistics.

The mean start and stop times from the first block are shown in Figure 2. As can be seen, mean start times for the single stimuli occurred prior to the associated criterion duration and stop times fell after the criterion duration. Similar to the peak functions, the start and stop times on compound trials fell in between the start and stop times from the single stimulus trials.

ANOVAs on the start times and stop times both showed significant effects of duration, $F(2,16)=55.034$ and 187.489 , respectively, $p<.001$. Post hoc comparisons confirmed that start and stop times for the compound cue were significantly different from those of the 10-s and 20-s cues (all $p<.001$ ). The coefficient of variation $(\mathrm{CV})$ was also computed for the start and stop times to ascertain whether there was greater within subject variability on compound trials. An ANOVA on the CVs of the start and stop times revealed an effect of duration, $F(2,16)=3.872, p<.05$ for the start times (start $\mathrm{CV}=0.49,0.56,0.47$ for $10 \mathrm{~s}$, compound, and $20 \mathrm{~s}$, respectively) and a slight trend toward a duration effect ( $p=.073$ ) for the stop times (stop $\mathrm{CV}=0.24,0.26,0.22$ for $10 \mathrm{~s}$, compound, and $20 \mathrm{~s}$, respectively). Post hoc analyses for the start times showed a trend for the compound CVs to be larger than those of the $10 \mathrm{~s}(p=.064)$ and $20 \mathrm{~s}(p=.061)$ cues. There were no main effects of stimulus modality, $F(1,8)=1.200,0.411,0.311$, 0.415 for start times, stop times, start time $\mathrm{CV}$, and stop time $\mathrm{CV}$, respectively; nor any Modality $\times$ Duration interactions, $F(2$, 16) $=0.638,0.811,1.497,0.180$ for start times, stop times, start time $\mathrm{CV}$, and stop time $\mathrm{CV}$, respectively; for any of the single trial analyses. In addition, there was a positive correlation between start and stop times on compound trials (mean $R^{2}=0.22$ ), which did not significantly differ from the correlations found for the 10-s $(0.29)$ or 20 -s trials $(0.32), F(2,16)=1.206, n s$.

When block was used as a factor in our analyses, a number of interactions emerged; however, the effects of duration remained significant, with few exceptions. For peak times, there was a Duration $\times$ Block $\times$ Modality interaction, $F(4,32)=2.728, p<$ .05 , and a strong trend toward a Duration $\times$ Modality interaction $(p=.056)$. Peak rates changed as a function of block, as indicated by a Block $\times$ Duration interaction, $F(2,16)=3.282, p<.05$. Post hoc analyses of peak rate for the compound cue revealed a significant reduction in response rate from Block 1 (0.96 resp/s) to Blocks $2(0.79 \mathrm{resp} / \mathrm{s})$ and $3(0.76 \mathrm{resp} / \mathrm{s}$; both $p<.01)$. All comparisons for normalized spreads were nonsignificant, as the main effect of duration reported above was reduced to a weak trend $(p=.08)$. The start times also showed a significant effect of 

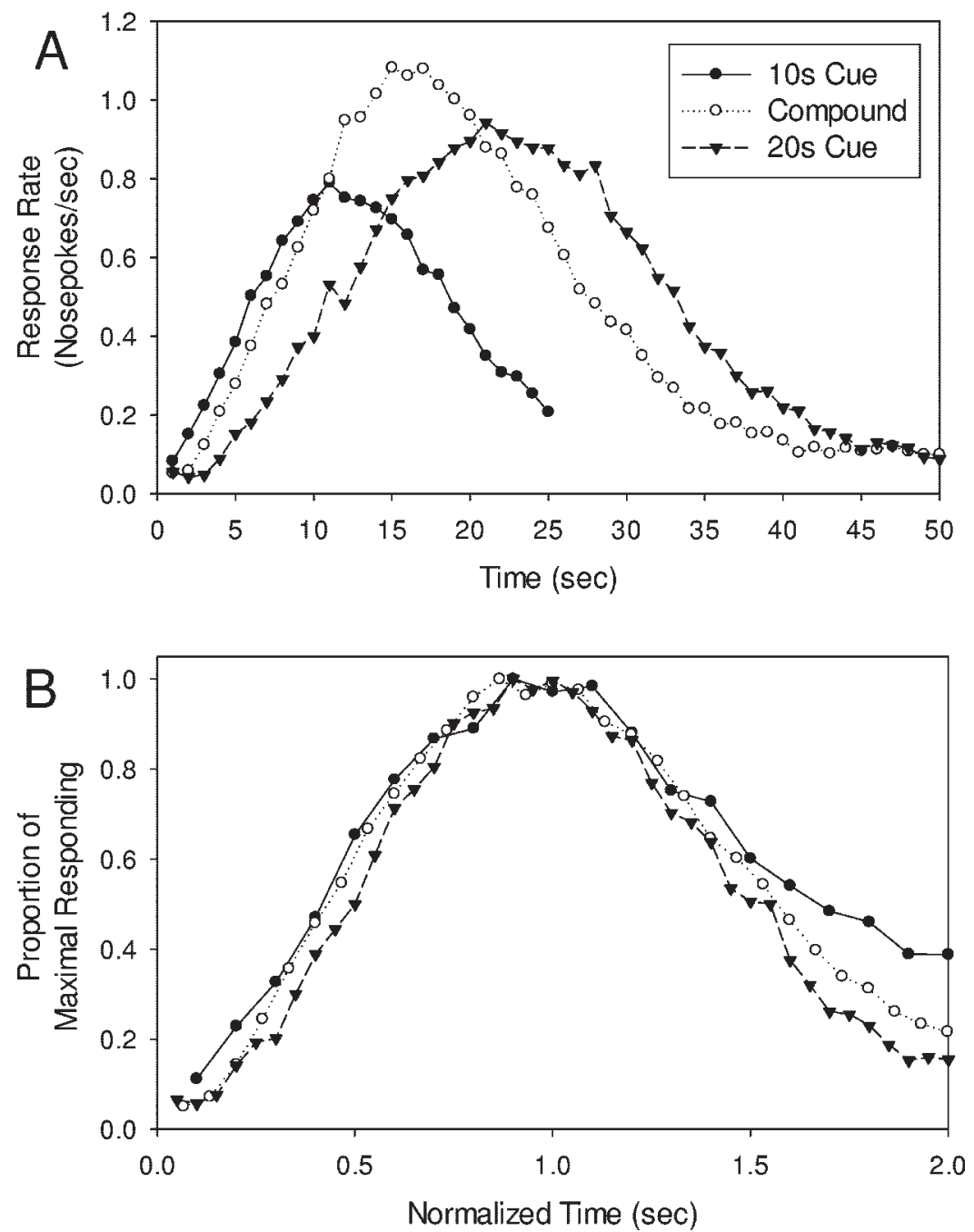

Figure 1. Panel A shows "peak" functions on 10-s alone, 20-s alone, and 10-s to 20-s compound stimulus trials. Rats were never reinforced on compound trials, yet showed a single, robust peak of activity at the midpoint between the two criterion durations. Panel B shows the peak functions after being normalized by their respective peak times. The data show that responding for the compound was roughly scalar, as the functions largely superimpose.

block, $F(2,16)=4.818, p<.05$; a Block $\times$ Duration interaction, $F(2,16)=3.429, p<.05$; and a weak trend toward a Duration $\times$ Block $\times$ Modality interaction $(p=.094)$. For the stop times, there was a Duration $\times$ Block $\times$ Modality interaction, $F(4,32)=$ $12.147, p<.001$; a trend toward an effect of block $(p=.062)$ and a weak trend toward a Duration $\times$ Modality interaction $(p=$ .088). There was no main effect of stimulus modality in any analysis with block as a factor (all $F_{\mathrm{s}}<1$ ). The start times and stop times are shown as a function of block and modality in Supplementary Figure 1.

\section{Discussion}

The present data clearly demonstrate that when rats are presented with stimuli that provide competing temporal information, they behave with respect to the average information provided by the cues. A somewhat similar pattern of results was reported in pigeons by Cheng and Roberts (1991). However, in that study, the pigeons showed very weak responding to the compound stimulus. As such, the only conclusion that could be drawn from those data was that the most probable time of response was somewhere in between the two programmed durations. In contrast, the current results demonstrate strong, peak-shaped responding at a time midway between the two reinforced durations. More important, the relative width of responding for the compound was roughly scalar when examining the mean functions, and was only slightly broader than scalar when examining the variability of the start and stop times. As such, the present data would not result from a simple additive process in which the output from multiple-independent estimates is brought together in behavior, which would lead to a peak in activity that begins around the short duration start time and ends around the long duration stop time. This interpretation is further supported by the positive correlation between start and stop 


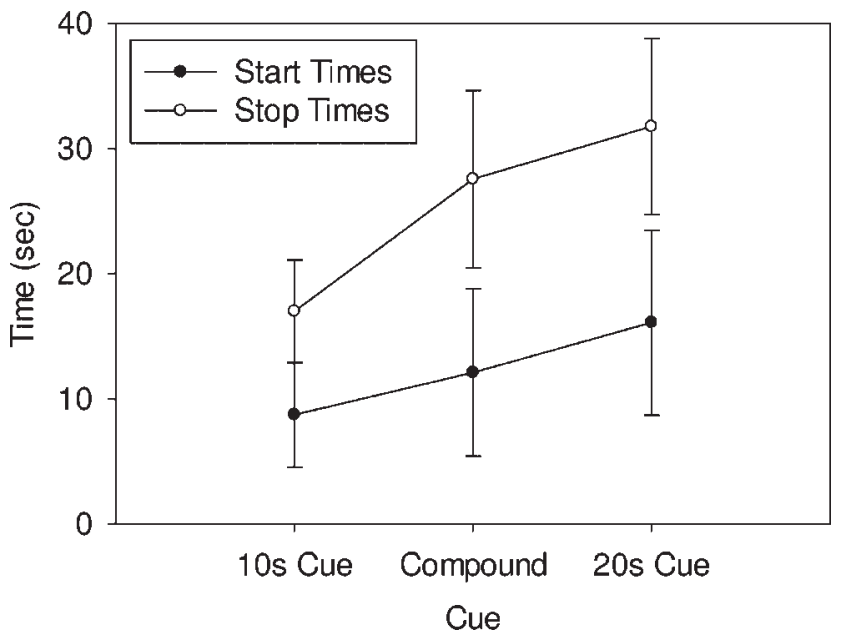

Figure 2. Start (filled) and stop (open) times from the single trial analyses for the 10-s alone, 20-s alone, and 10-s to 20-s compound stimulus trials. Error bars reflect the average standard deviation of these statistics within each subject. The start and stop times for the compound trials occurred around the midpoints of those from the 10-s and 20-s trials.

times seen on compound trials, which would be unlikely if responding for the compound was produced by independently responding for the short and long cues in an additive manner. Similarly, these data are inconsistent with a process in which only one of the cues is selected for use on each trial, which would lead to substantially larger variability of start and stop times than that found here. Rather, temporal control appears to result from normal temporal processes working with an average computed from discrepant temporal memories.

Given these data showing a single peak of responding with characteristics consistent with timing a single average duration, one obvious question is what component of the timing system is being averaged to create this behavior, as current theories of timing do not make predictions about timing of two concurrent stimuli. One possible explanation, given the typical finding of lower associative strength for longer durations (Jennings, Bonardi, \& Kirkpatrick, 2007), is that rats time only the short duration on compound trials (e.g., Meck \& Church, 1984), with the presence of the long cue diminishing the associative value of the short cue, thereby slowing clock speed and leading to a peak time to the right of the short duration. Alternatively, perhaps the rats timed the longer duration on compound trials, but with a faster clock speed due to the presence of the short cue. As we attempted to equate reinforcement densities in the present experiment by assigning different probabilities of reinforcement to the short and long cues, and as peak rates did not differ between durations, we think this explanation is unlikely. However, we did find a greater proportion of omitted trials for the short cue than either the long or compound cues, suggesting that local, rather than session wide, reinforcement densities, may be computed by the rats. As such, explanations based on differential associative strengths of the cues cannot be ruled out.

An alternative possibility, following from the information processing framework of scalar expectancy theory (Gibbon \& Church, 1984), is that two memory samples, one for each duration cue, are drawn at the onset (Meck, 1984) of the compound stimulus, and these discrepant memories are averaged to produce a single expectation. Alternatively, rats may not be averaging temporal memories, per se, but rather clock speeds. The behavioral theory of timing (Killeen \& Fetterman, 1988), proposed that timing is carried out by progressing through a sequence of behavioral states, with the transitions between states signaled by a pacemaker pulse. In this theory, the different reinforcement densities of each signal lead to different clock speeds, such that the time of responding is inversely proportional to the speed of the pacemaker. As such, it may be the case that rats average the clock speeds for the two durations. However, it should be noted that in this theory, the stimulus-clock speed relationship is the referent temporal memory. As such, although we cannot determine whether it is the memory samples for the anticipated times of reinforcement or the clock speeds associated with each cue that are being averaged, some form of temporal memory averaging appears to occur.

Although we believe it is premature at this point to draw any conclusions regarding the type of averaging being done on compound trials, we computed the arithmetic, geometric and harmonic means using the observed data points from the 10-s and 20-s trials and compared those averages to the observed responding on compound trials. We also calculated the weighted versions of these means using the probability of reinforcement for short and long trials as weights. Peak time was best predicted by a weighted geometric mean, as was the stop time. In contrast, the start time was best predicted by the unweighted geometric mean. It is interesting to note that when Meck, Komeily-Zadeh, and Church (1984) trained rats using one duration and then subsequently shifted reinforcement to a later duration, the rats initially responded in a manner consistent with the geometric mean of the two durations.

A somewhat similar form of temporal averaging has been shown in unmedicated Parkinson's disease patients when tested sequentially with multiple durations (Malapani, Deweer, \& Gibbon, 2002; Malapani et al., 1998). By removing medication during either training or testing, Malapani and colleagues were able to demonstrate that the reproductions of durations "migrated" toward one another as a result of impaired functioning during the testing phase of the experiment, suggesting that aspects of temporal memory retrieval were disrupted by decreased dopaminergic tone, rather than alteration of function during the encoding phase. Although the current results show a similar averaging of durations, it should be noted that the migrated reproductions of the Parkinson's patients were not scalar, and thus these patients are likely to have additional alterations to their timing process (Shea-Brown, Rinzel, Rakitin, \& Malapani, 2006). It remains to be seen whether the current averaging is mediated by similar neurobiological substrates as those disrupted by the disease.

Averaging of perceptual and memory/decision substrates has also been reported for other domains. Visual motion direction identification has been shown to result from vector averaging of neural activity (Lisberger \& Ferrera, 1997), as has motor control (Georgopoulos, Schwartz, \& Kettner, 1986). In more complex cognitive domains, pigeons, when presented with multiple landmarks that have previously provided congruent information regarding the location of food, will search in a location associated with a weighted average of these cues when the information provided becomes discrepant (Cheng, 1989). In another experi- 
ment, subjects were trained that correct performance results from searching at both a specific location and a specific time in reference to a moving landmark. When the speed of the landmark movement is varied, thereby putting time and space into conflict, both pigeons and humans search at a time and place predicted by the average of these two information sets (Cheng et al., 1996). However, the temporal aspects of responding in this experiment were not scalar, and the time at which responding began did not vary with changes in movement rate, suggesting that the timespace averaging is occurring at the performance end of the computation, rather than in memory as seems to occur here. Taken together with the current results, it appears that averaging of discrepant information is a coding strategy used across the inputoutput domains of a variety of modalities, and may play a strong role in a variety of behaviors.

\section{References}

Aydin, A., \& Pearce, J. M. (1997). Some determinants of response summation. Animal Learning and Behavior, 25, 108-121.

Bateson, M. (2003). Interval timing and optimal foraging. In W. H. Meck (Ed.), Functional and neural mechanisms of interval timing (pp. 113141). Boca Raton, FL: CRC.

Boisvert, M. J., \& Sherry, D. F. (2006). Interval timing by an invertebrate, the bumble bee bombus impatiens. Current Biology, 16, 1636-1640.

Brunner, D., Kacelnik, A., \& Gibbon, J. (1992). Optimal foraging and timing processes in the starling Sturnus vulgaris: Effect of intercapture interval. Animal Behaviour, 44, 597-613.

Cheng, K. (1989). The vector sum model of pigeon landmark use. Journal of Experimental Psychology: Animal Behavior Processes, 15, 366-375.

Cheng, K., \& Roberts, W. A. (1991). Three psychophysical principles of timing in pigeons. Learning and Motivation, 22(1-2), 112-128.

Cheng, K., Spetch, M. L., \& Miceli, P. (1996). Averaging temporal duration and spatial position. Journal of Experimental Psychology: Animal Behavior Processes, 22, 175-182.

Church, R. M. (1997). Timing and temporal search. In C. M. Bradshaw \& E. Szabadi (Eds.), Time and behaviour: Psychological and neurobehavioural analyses (Vol. 120, pp. 41-78). Amsterdam: Elsevier.

Church, R. M., Meck, W. H., \& Gibbon, J. (1994). Application of scalar timing theory to individual trials. Journal of Experimental Psychology: Animal Behavior Processes, 20, 135-155.

Emurian, H. H., \& Weiss, S. J. (1972). Compounding discriminative stimuli controlling free-operant avoidance. Journal of the Experimental Analysis of Behavior, 17, 249-256.

Fairhurst, S., Gallistel, C. R., \& Gibbon, J. (2003). Temporal landmarks: Proximity prevails. Animal Cognition, 6, 113-120.

Gallistel, C. R., \& Gibbon, J. (2000). Time, rate, and conditioning. Psychological Review, 107, 289-344.

Georgopoulos, A. P., Schwartz, A. B., \& Kettner, R. E. (1986). Neuronal population coding of movement direction. Science, 233, 1416-1419.

Gibbon, J., \& Church, R. M. (1984). Sources of variance in an information processing theory of timing. In H. L. Roitblat, T. G. Bever, \& H. S. Terrace (Eds.), Animal cognition. Hillsdale, NJ: Erlbaum.

Gibbon, J., Fairhurst, S., \& Goldberg, B. (1997). Cooperation, conflict and compromise between circadian and interval clocks in pigeons. In E. C. M. Bradshaw \& E. E. Szabadi (Eds.), Time and behaviour: Psychological and neurobehavioural analyses (pp. 329-384). Amsterdam: Elsevier.

Henderson, J., Hurly, T. A., Bateson, M., \& Healy, S. D. (2006). Timing in free-living rufous hummingbirds, Selasphorus rufus. Current Biology, 16, 512-515.

Higa, J. J. (1997). Dynamics of temporal control in rats: The effects of a brief transition in interval duration. Behavioural Processes, 40, 223 229.

Jennings, D. J., Bonardi, C., \& Kirkpatrick, K. (2007). Overshadowing and stimulus duration. Journal of Experimental Psychology: Animal Behavior Processes, 33, 464-475.

Kehoe, E. J. (1986). Summation and configuration in conditioning of the rabbit's nictitating membrane response to compound stimuli. Journal of Experimental Psychology: Animal Behavior Processes, 12, 186-195.

Killeen, P. R., \& Fetterman, J. G. (1988). A behavioral theory of timing. Psychological Review, 95, 274-295.

Kirkpatrick, K., \& Church, R. M. (2000). Stimulus and temporal cues in classical conditioning. Journal of Experimental Psychology: Animal Behavior Processes, 26, 206-219.

Lisberger, S. G., \& Ferrera, V. P. (1997). Vector averaging for smooth pursuit eye movements initiated by two moving targets in monkeys. Journal of Neuroscience, 17, 7490-7502.

Malapani, C., Deweer, B., \& Gibbon, J. (2002). Separating storage from retrieval dysfunction of temporal memory in Parkinson's disease. Journal of Cognitive Neuroscience, 14, 311-322.

Malapani, C., Rakitin, B., Levy, R., Meck, W. H., Deweer, B., Dubois, B., et al. (1998). Coupled temporal memories in Parkinson's disease: A dopamine-related dysfunction. Journal of Cognitive Neuroscience, 10, 316-331.

Matell, M. S., Bateson, M., \& Meck, W. H. (2006). Single-trials analyses demonstrate that increases in clock speed contribute to the methamphetamine-induced horizontal shifts in peak-interval timing functions. Psychopharmacology, 188, 201-212.

McLaren, I. P., \& Mackintosh, N. J. (2002). Associative learning and elemental representation: II. Generalization and discrimination. Animal Learning and Behavior, 30, 177-200.

Meck, W. H. (1984). Attentional bias between modalities: Effect on the internal clock, memory, and decision stages used in animal time discrimination. Annals of the New York Academy of Sciences, 423, 528 541.

Meck, W. H., \& Church, R. M. (1984). Simultaneous temporal processing. Journal of Experimental Psychology: Animal Behavior Processes, 10, $1-29$.

Meck, W. H., Komeily-Zadeh, F. N., \& Church, R. M. (1984). Two-step acquisition: Modification of an internal clock's criterion. Journal of Experimental Psychology: Animal Behavior Processes, 10, 297-306.

Miller, L. (1971). Compounding of discriminative stimuli from the same and different sensory modalities. Journal of the Experimental Analysis of Behavior, 16, 337-342.

Miller, R. R., \& Barnet, R. C. (1993). The role of time in elementary associations. Current Directions in Psychological Science, 2, 106-111.

Rakitin, B. C., Gibbon, J., Penney, T. B., Malapani, C., Hinton, S. C., \& Meck, W. H. (1998). Scalar expectancy theory and peak-interval timing in humans. Journal of Experimental Psychology: Animal Behavior Processes, 24, 15-33.

Shea-Brown, E., Rinzel, J., Rakitin, B. C., \& Malapani, C. (2006). A firing rate model of Parkinsonian deficits in interval timing. Brain Research, 1070, 189-201.

Weiss, S. J. (1972). Stimulus compounding in free-operant and classical conditioning: A review and analysis. Psychological Bulletin, 78, 189208.

Wiener, M., Magaro, C. M., \& Matell, M. S. (2008). Accurate timing but increased impulsivity following excitotoxic lesions of the subthalamic nucleus. Neuroscience Letters, 440, 176-180.

Received December 3, 2007

Revision received July 25, 2008 Accepted July 29, 2008 